\section{Biomechanical Analysis of the Pelvis Angular Excursion in Sagittal Plane in Response to Asymmetric Leg Loading Tasks in Females with and without Non- specific Chronic Low Back Pain}

\author{
Narges Meftahi, ${ }^{1,2}$, Fahimeh Kamali,2*๑, Mohamad Parni- \\ anpour 3 , Mehrdad Davoudi ${ }^{4}$
}

\begin{abstract}
Background: Controlling pelvic excursions is the focus of stabilization exercises such as legs loading tasks in rehabilitation of non-specific chronic low back pain (NSCLBP) patients. Progression of these exercises is based on the ability to perform tasks with minimal sagittal pelvic excursions. In spite of emphasis on minimizing pelvic motions, no previous studies have investigated kinematic analysis of the pelvic excursions during leg loading exercises in NSCLBP patients.
\end{abstract}

Objective: This study aims to investigate the sagittal pelvis excursion during performing asymmetric leg loading tasks in individuals with and without NSCLBP.

Material and Methods: In this cross-sectional study, kinematic data were collected from 15 NSCLBP patients and 15 asymptomatic participants by a motion analysis system during right and left leg loading tasks with 2 levels of difficulty. Pelvis segments were modeled using Visual3D motion analysis software. Maximum pelvic excursion in the sagittal plane was calculated during each task. Mixed model analysis of variances (group, task difficulty level, side) was performed for statistical analysis.

Results: The maximum sagittal pelvic excursion values of all tasks in NSCLBP were smaller than those in the control group; however, no significant main effects and interactions were found between two groups.

Conclusion: These results suggest that NSCLBP patients completed loading tasks without differences in sagittal pelvic excursions as compared to controls. Assessment of NSCLBP patients only based on pelvic angular excursion may not be sufficient for clinical decision making. Furthermore, asymptomatic individuals may need to practice for controlling pelvic excursion during leg loading exercises similar to the CLBP patients. Citation: Meftahi N, Kamali F, Parnianpour M, Davoudi M. Biomechanical Analysis of the Pelvis Angular Excursion in Sagittal Plane in Response
to Asymmetric Leg Loading Tasks in Females with and without Non-specific Chronic Low Back Pain. J Biomed Phys Eng. 2021;11(3):367-376. to Asymmetric Leg Loading

Keywords

Low Back Pain; Pelvis; Biomechanical Phenomena; Exercise Therapy

\section{Introduction}

hronic low back pain (CLBP) is a common health issue, affecting significantly functional ability and quality of life of a person. Lack of an identified specific pathology or origin puts most of
${ }^{1}$ PhD, Physical Therapy

Department, School of

Rehabilitation Sciences,

Shiraz University of

Medical Sciences, Shi-

raz, Iran

${ }^{2}$ PhD, Rehabilitation

Sciences Research

Center, Shiraz University

of Medical Sciences,

Shiraz, Iran

${ }^{3} \mathrm{PhD}$, Department of

Mechanical Engineering,

Sharif University of Tech-

nology, Tehran, Iran

${ }^{4} \mathrm{MSc}$, Department of

Mechanical Engineering,

Sharif University of Tech-

nology, Tehran, Iran

*Corresponding author:

Fahimeh Kamali

Physical Therapy Depart

ment, School of Rehabil-

itation Sciences, Shiraz

University of Medical

Sciences, Abiverdi 1 Ave,

Chamran Blvd, Shiraz,

Fars, Iran

E-mail: fahimehkamali@

hotmail.com

Received: 16 May 2018

Accepted: 20 June 2018 
these patients in a large heterogeneous group named non-specific CLBP (NSCLBP) [1, 2]. A popular choice in the rehabilitation regimes of NSCLBP is dynamic stability exercise protocols because evidence supports the relationship between lumbo-pelvic instability and CLBP [3-8]. Measuring different electromyographic, kinetic and kinematics variables of these stability exercise protocols would add clinicians information about characteristics of these exercises leading to make better choices for designing a rehabilitation program for NSCLBP patients. Asymmetric leg-loading task is a commonly used dynamic stability exercise in rehabilitation of LBP. It serves both as a test to evaluate function and an exercise advancement. It includes a primary abdominal hallowing maneuver fallowed by a set of legloading perturbations with emphasis on maintaining lumbo-pelvic neutral position during the protocol. It has different levels of difficulty through, providing challenges to neuromuscular system [9-12]. Since the specific emphasis in this protocol is minimizing and controlling lumbo-pelvic motions against perturbations caused by various movements, it is also valuable to quantify pelvic motion during these exercises in NSCLBP. Pelvic kinematics in LBP patients have been assessed during various activities, including stair-climbing [13], walking [14, 15], running [14], trunk axial rotation [16], active knee flexion [17], active hip lateral rotation in prone $[17,18]$ and trunk forward bending and backward return [19] in previous investigations. Biomechanical studies of pelvic kinematics during specific stabilization exercise such as leg-loading task, however, are not usual. Asymmetric leg-loading task has recently attracted the researchers in the field of electromyography to evaluate muscle activation patterns during this task [10, 11, 20-24]. Pelvic displacements were often controlled visually in these studies. However, only two of these investigations have objectively measured the kinematics of the pelvic segment during the leg-loading tasks [22, 24]. Hubley-Kozey et al. studied abdominal muscle activation patterns and angular displacement of the pelvis during right leg-loading task in younger and older healthy adults. No significant differences of pelvic angles were reported between these two groups [22]. Furthermore, Moreside et al. identified less pelvic angular displacements in participants, who were recovered from a recent $(<12 \mathrm{wk})$ low back injury compared to non-low back injury control group. They also reported that low back injured group were significantly older than control group [24]. Accordingly, there is a limited amount of literature regarding pelvic biomechanics during leg-loading task. Furthermore, the minimal available evidence is limited to the asymptomatic individuals $[22,24]$. To our knowledge, pelvic kinematics during asymmetric leg-loading tasks in NSCLBP has not been addressed in the previous studies. Therefore, the purpose of the present study was to compare pelvic kinematics between NSCLBP patients and an asymptomatic control group while performing leg-loading tasks. The legloadings in these tasks include flexion and extension of the knee and hip joints, which occurred in the sagittal plane. Therefore, it can be reasonably stated that the sagittal plane is probably the main motion plane affected in this task. The focus of the present study was on assessing sagittal plane pelvis excursions. Considering the higher incidence of LBP in females [25] and the reduction of the effects associated with gender on the kinematics [2628 ], only female participants were recruited in the present study.

Capturing biomechanical data of the pelvic motion during performing a stabilization exercise would provide an objective method of assessment with more precision than the subjective visual monitoring of the pelvis at the clinical level. Kinematic analysis of the pelvis angular excursion may help to determine whether the emphasis on minimizing pelvic 
motions for correctly performing stabilization exercise is valuable in clinical settings.

\section{Material and Methods}

\section{Participants}

In this cross-sectional study, two groups of participants consisted of 15 female patients with NSCLBP and 15 asymptomatic females volunteered. The patients were selected among females referred by orthopedists to physical therapy clinics with CLBP diagnosis whereas the non-LBP controls were recruited via printed and electronic advertisements. All participants were matched based on age, weight and height (Table 1) and received a clear description of the investigation prior to participation in this research. They completed and signed an informed consent form approved by the Shiraz University of Medical Sciences ethics committee (No: 11173).

Individuals were eligible for the study if they were between 18 and 50 years old. The inclusion criteria for the control group were as follows: having no history of back pain that resulted in drug therapy or absence from the workplace, having no history of any incidence of back pain that lasts for more than three months. The patients were included in

Table 1: Group descriptive data mean \pm SD

\begin{tabular}{cccc} 
Group & Control & NSCLBP & P-Value \\
\hline Age (years) & $26.80 \pm 3.71$ & $29.70 \pm 3.68$ & 0.186 \\
\hline Height $(\mathrm{m})$ & $1.62 \pm .07$ & $1.58 \pm .04$ & 0.255 \\
\hline Weight $(\mathrm{kg})$ & $56.26 \pm 8.08$ & $60.67 \pm 6.1$ & 0.359 \\
\hline BMl & $21.42 \pm 2.05$ & $24.34 \pm 2.65$ & 0.078
\end{tabular}

BMI: body mass index; NSCLBP; non-specific chronic low back pain the NSCLBP group if they had permanent or alternate back pain for more than three months and the first attack was at least six months ago [29]. In addition, the severity of the pain was such that it requires treatment (non-surgical) or absence from work or rest at home. Patients were not on leave because of their back pain on the test day. The following exclusion criteria were applied in low back pain due to trauma, radicular pain to lower limbs, spondylolisthesis, history of any fracture or surgery of the trunk, spine or lower extremity, any spinal or lower limbs' deformity, systemic diseases such as rheumatoid disease and diabetes, taken any sedative/analgesic medicine or opioids and alcohol in the past 48 hours, alcohol or drug addiction and rehabilitation program, including spinal stabilization exercises in the last year, any orthopedic/neurologic or sensory disorders and inability to perform tests during study due to increased pain. It should be noted that none of the participants should have previous familiarity with the loading tasks. In addition, the pain score of the all patients should be 3 and lower based on numeric rating scale during the test session.

\section{Data collection}

A motion analysis system with 8 high speed cameras (Proreflex, Qualisys Track Manager Ltd., Gothenburg, Sweden) was used to record the kinematic data at a sampling rate of 100 $\mathrm{Hz}$ while doing the leg-loading tasks. Infraredretroreflective markers were attached bilaterally on the iliac crests, anterior superior iliac spines, greater trochanters, medial and lateral femoral condyles, medial and lateral malleoli, heels, first, second and fifth metatarsal heads, fifth metatarsal base. Moreover, 4 clusters (each consists of 4 markers) were attached on anterolateral surfaces of thigh and shank in both right and left sides. A static trial was recorded before each task. For leg-loading trials, the calcanei markers were then removed.

Participants instructed to perform the asym- 
metric leg-loading protocol. This task was performed separately for the left and right lower extremity. The participant was placed in the supine position with both knees at $90^{\circ}$ of flexion, as measured with a plastic standard goniometer, and feet flat on an examination couch for the starting position of each level. Prior to carry out of tasks, participants were asked to do an abdominal hollowing maneuver as a preliminary step and to maintain it during the task. To test the right lower extremity, following steps were done: The participant lifted the right foot off the couch and continued raising it until the right thigh touched a frame. The right hip joint was placed at $90^{\circ}$ of flexion in this position. The left leg was then lifted to the same position (leg-lifting phase). In the next step, only the right hip and knee were fully extended and then flexed back until the right thigh came into contact with the frame again (leg-extension/loading phase). The left and then the right lower extremity were subsequently lowered to the starting position (leglowering phase). To test the left lower extremity, the similar protocol was done. The height of the frame changed according to the length of the participant's thigh. The total task was completed within 8 seconds. The participant was asked to perform each steps by hearing a sound from the metronome. We assessed the leg-loading task with 2 levels of difficulty. These two levels differ from each other only in the leg-extension phase. In level 1, leg-extension phase was done while the participant slid her heel lightly on the examining couch. However, leg-extension was carried out without the contact of the heel with the couch in level 2 [21-24]. Therefore, each participant performed 4 tasks: right heel slide (RHS), right no-heel slide (RNHS), left heel slide (LHS), left no-heel slide (LNHS). Three trials, with at least 2-minute rest between trials, were recorded for each task. The order of tasks were randomly assigned. Before recording the test trials, participants were allowed to practice the tasks until they can perform them correctly. Prior to data collection, the participants were asked to be relaxed $2 \mathrm{~s}$ in the starting position to record the static trial as a reference.

Data analysis

All data processing was done through $\mathrm{Vi}$ sual3D motion analysis software (C-Motion, Rockville, MD, USA). Raw data were filtered by a low-pass, fourth order Butterworth filter with a cut off frequency of $6 \mathrm{~Hz}$. By the marker set in Visual3D software, one pelvis segment, two thigh segments, two shank segments, and two foot segments were modeled. The start and end time of each task was determined by using high pass algorithm (HPA) method and also confirmed visually for possible inaccurate phase detection because of the lost marker data. The start time was the moment when the foot began to raise from the bed and the end time was the moment when the same foot began to touch the bed. The pelvic angle in the sagittal plane (anterior/posterior tilt) was computed at each time point during each task. The initial static starting position was regarded as the reference posture. Maximum pelvic excursion was calculated from the difference between the peak value of pelvic angle in each trial and its corresponding value at the reference posture. The average value of three trials was considered for statistical analyses in each task.

\section{Statistical analysis}

All statistical data analyses were done in IBM $^{\circledR}$ SPSS, version 21.0 (IBM Corp, Armonk, NY, USA) and the level of significance was considered with $\mathrm{P}$-value smaller than 0.05 . The normal distribution of data was checked by Shapiro-Wilk test. Independent sample t-test was applied to compare the demographic data between 2 groups. One set of mixed model ANOVA tests was performed for main and interaction effects of group (with and without LBP), side (left and right leg- 
loading tasks), and task difficulty level (leg extension phase with and without heel slide) on the pelvis angular excursions during LHS, LNHS, RHS and RNHS tasks. The interaction effect was removed from the model if it was not significant.

\section{Results}

There were no significant differences between 2 groups with respect to the demo- graphic characteristics (Table 1). Participants in 2 groups were compared with regard to maximum excursion of the pelvis in the sagittal plane. The mean maximum angles for each group, each level of task difficulty and each side are indicated in Table 2. Although the maximum pelvic angular excursion in NSCLBP was smaller than these values in control group (Table 2), there were no significant main effects of group, task difficulty level

Table 2: Descriptive data (mean \pm SD) of maximal pelvic rotation range of motion in sagittal plane

\begin{tabular}{ccccl} 
Group & LHS & LNHS & RHS & RNHS \\
\hline Control & $20.8 \pm 10.1$ & $22.7 \pm 14.7$ & $23.2 \pm 20.9$ & $23.5 \pm 18.2$ \\
\hline NSCLBP & $16.0 \pm 9.2$ & $19.0 \pm 10.8$ & $14.4 \pm 7.3$ & $13.7 \pm 8.4$
\end{tabular}

NSCLBP: non-specific chronic low back pain; LHS: left heel slide; LNHS: left no-heel slide; RHS: right heel slide; RNHS: right no-heel slide.

and side on the pelvis excursion. In addition, no significant interaction effects of independent variables on the pelvis excursion were observed (Table 3).

\section{Discussion}

The purpose of the present study was to investigate pelvic kinematics in the sagittal plane during performing asymmetric legloading tasks with two levels of difficulty. We compared NSCLBP patients with an asymptomatic control group. The results of the current study demonstrated that there were no significant differences in the sagittal plane maximum rotational excursion of the pelvis between these 2 groups during right and left leg-loading tasks with two levels of difficulty.

In terms of similarity to the studied population of the present study, some prior investigations are available on the pelvic kinematics in patients with LBP. However, different motion tasks such as stair-climbing, walking, running, trunk axial rotation and active hip lateral rotation in patients with CLBP, active knee flexion and hip lateral rotation in prone in CLBP associated with rotation-related sport and trunk forward bending and backward return in acute LBP were studied [13-19]. Some of these previous studies have shown significant differences between CLBP and control groups in the pelvis angular excursion values. However, others have not reported these differences, which were consistent with the findings of the present study. Lee et al. have demonstrated that CLBP patients had no significant differences in the $3 \mathrm{D}$ pelvic motion as compared to healthy controls during stair climbing. They have reported that the only different kinematic variable between CLBP and control groups was the lumbar spinal flexion/ extension range of motion. Thoracic motions were also not different between groups. They 
Table 3: Statistics for the effects of group (with and without low back pain (LBP)), (legloading with and without heel slide) and side (right leg and left leg) on pelvic ranges of rotation in the sagittal plane

\begin{tabular}{ccc} 
& \multicolumn{2}{r}{ Pelvic Rotation } \\
\hline \multirow{2}{*}{ Group } & $\mathrm{F}$ & 1.563 \\
\cline { 2 - 3 } Difficulty level & $\mathrm{P}$ & 0.221 \\
\hline Side & $\mathrm{F}$ & 2.816 \\
\cline { 2 - 3 } & $\mathrm{P}$ & 0.096 \\
\hline \multirow{2}{*}{ Group $\times$ Difficulty level } & $\mathrm{F}$ & 0.060 \\
\cline { 2 - 3 } & $\mathrm{P}$ & 0.807 \\
\hline \multirow{2}{*}{ Group $\times$ Side } & $\mathrm{P}$ & 0.013 \\
\hline \multirow{2}{*}{ Difficulty level $\times$ Side } & $\mathrm{F}$ & 2.987 \\
\cline { 2 - 3 } & $\mathrm{P}$ & 0.086 \\
\hline \multirow{2}{*}{ Group $\times$ Difficulty level $\times$ Side } & $\mathrm{F}$ & 1.151 \\
\cline { 2 - 3 } & $\mathrm{P}$ & 0.285 \\
\cline { 2 - 3 } & $\mathrm{P}$ & 0.799 \\
\hline
\end{tabular}

stated that the reason for these results could be the most direct and localize effect of LBP on the lumbar spine in stair-climbing [13]. Taniguchi et al. also have observed no significant differences in rotational range of motions of the spine, thorax and pelvis between CLBP and asymptomatic groups at maximum rotation position and $50 \%$ rotation position in transverse plane. They have depicted that the difference between two groups was at spine/ pelvis ratio (an index of movement pattern) which was significantly greater than in CLBP patients compared to control group. They conclude that the most affected parameter by the CLBP was the movement pattern not maximum rotation range of motion [16]. Lamoth et al. stated that usual kinematic measures such as range of motion might mask the differences between LBP patients and control group; however, coordination measures could distinguish differences between them. They showed that although there was no significant difference in the transverse rotation of the pelvic and thoracic segments, the coordination between these two segments had changed [15]. Although the motion tasks in these previous investigations were not the same as the present study, their findings suggested that pelvic excursions in CLBP patients could remain unchanged, consistent with the results of the current study. Similar to the changes in the movement pattern in CLBP during different tasks, HubleyKozey and Vezina have reported deficiency in temporal activation patterns of trunk muscles during leg-lifting and leg-lowering tasks in CLBP patients. They did not report any data on pelvic motions [20].

In terms of similarity to the studied task in the present study, only two studies have been conducted to assess pelvic kinematics during leg-loading tasks $[22,24]$. Their findings are not directly comparable because these studies examined asymptomatic individuals. Moreside et al, demonstrated that pelvic motions in individuals who recovered from an acute first injury to the low back were less than asymptomatic ones. Compared to the current study, the cause of LBP in their participants was a specific injury and they were not in chronic phase of LBP. They also had a significant difference in age with the control group [24]. Not only the condition of a person in chronic phase differs from acute or subacute phase [30], but also the age and gender is expressed as a factor affecting pelvic kinematics [19, 26-28, 31]. The presence of the changes in the central nervous system and the psychological factors associated with chronicity distinguishes this condition from acute and subacute conditions [32-34]. To minimize the effect of age and gender, our participants were age-matched females. Given the above issues, the results of the present study cannot be compared with 
these previous studies.

The asymptomatic control group in the current study did not show significant differences in maximum pelvic excursion as compared to the CLBP groups. There is evidence that showed that all healthy asymptomatic people are not necessarily able to do stabilization exercises such as leg-loading task with minimal pelvic motion. Elia et al. found that skill acquired through adequate familiarity and practice is an effective factor in the ability of an individual to minimize the pelvic motion during a stabilization exercise. As they reported, the pelvic range of motion in expert individuals was smaller than that in beginners who were unfamiliar with stabilization training [35]. Hubley-Kozey et al. attributed the lack of significant differences in pelvic angular displacement between healthy younger and older adults to the sufficient training sessions considered before the test sessions. They reported these practice sessions were useful to train the participants to do the task with minimal pelvic motion [22]. The leg-loading task was considered as an inclusion criteria in the present study because it was reported that having a certain amount of familiarity and practice would result in a coordinated pattern of movement, which affect the muscle activation and kinematics [35]. However, in the present study, the participants received detailed instruction and were allowed to practice before the test as long as they had learned the task.

Another possibility is that the asymptomatic group in this study had masked and had underlying dysfunction of muscles controlling the stability of the pelvis like trunk core muscles. In this situation, the ability of neuromuscular system to sufficiently control the motion of lumbo-pelvic region during leg-loading may decrease. Hubley-Kozey et al. classified healthy people into stable and unstable groups to evaluate abdominal muscle activation as well as pelvic motion during leg-loading task. Their criteria for group assignment were ac- cording to the participants' capability to minimize lumbo-pelvic motion during performing the task. Despite the limitation of these visual criteria, some differences were reported in the waveforms between these groups. They showed that despite being asymptomatic, muscle activation patterns might be altered [23].

The current study has some potential limitations. First, we investigated the pelvis excursion during a stability exercise. Generalization of these findings for daily activities is not known at this time. Second, the pelvic kinematic measurements were limited to the sagittal plane in the present study. Since the leg loadings in this task are composed of flexion and extension of the knee and hip joints, it can be reasonably stated that the sagittal plane is probably the main motion plane affected in this task. The third limitation states that the generalizability of the present study data is limited to the females. It is suggested examining the differences between men and women as well as other motion planes in future studies. Investigation of the pelvis movement pattern during loading tasks is also valuable.

\section{Conclusion}

This study compared the angular excursion of the pelvis in sagittal plane during leg-loading tasks in NSCLBP patients and asymptomatic control individuals. Our findings showed no significant differences in maximal pelvic excursion in sagittal plane between these groups. These findings suggest that the assessment of NSCLBP patients only based on kinematic variables such as angular excursion may be not sufficient for clinical decision-making. Furthermore, asymptomatic individuals may need to practice for controlling pelvic excursion during leg-loading stabilization exercise similar to the CLBP patients. Further research is required to investigate the movement pattern and coordination measures in NSCLBP patients. 


\section{Acknowledgment}

The authors would like to acknowledge scientific and financial support from Shiraz University of Medical Sciences, the Deputy for Research Affairs and the School of Rehabilitation Sciences. The present article was extracted from the $\mathrm{PhD}$ thesis written by Narges Meftahi and financially supported by Shiraz University of Medical Sciences Grant No. 11173.

\section{Conflict of Interest}

None

\section{References}

1. Hartvigsen J, Hancock MJ, Kongsted A, Louw Q, Ferreira ML, Genevay S, Hoy D, Karppinen J, Pransky G, Sieper J, Smeets RJ, Underwood M. What low back pain is and why we need to pay attention. The Lancet. 2018;391(10137):2356-67. doi: 10.1016/ S0140-6736(18)30480-X.

2. WHITE III AA, GORDON SL. Synopsis: workshop on idiopathic low-back pain. Spine. 1982;7(2):141-9.

3. Chung SH, You YY, Lee HJ, Sim SH. Effects of stabilization exercise using flexi-bar on functional disability and transverse abdominis thickness in patients with chronic low back pain. Journal of Physical Therapy Science. 2018;30(3):400-4. doi: 10.1589/jpts.30.400.

4. Russo M, Deckers K, Eldabe S, Kiesel K, Gilligan C, Vieceli J, Crosby P. Muscle control and non-specific chronic low back pain. $\mathrm{Neu}$ romodulation: Technology at the Neural Interface. 2018;21(1):1-9. doi: 10.1111/ner.12738.

5. Paungmali A, Joseph LH, Punturee K, Sitilertpisan P, Pirunsan U, Uthaikhup S. Immediate effects of core stabilization exercise on $\beta$-endorphin and cortisol levels among patients with chronic nonspecific low back pain: a randomized crossover design. Journal of Manipulative and Physiological Therapeutics. 2018;41(3):181-188. doi: 10.1016/j. jmpt.2018.01.002.

6. Shahvarpour A, Gagnon D, Preuss R, Henry SM, Larivière C. Trunk postural balance and low back pain: reliability and relationship with clinical changes following a lumbar stabilization exercise program. Gait \& Posture. 2018;61:375-381. doi: 10.1016/j.gaitpost.2018.02.006.

7. Ko KJ, Ha GC, Yook YS, Kang SJ. Effects of 12-week lumbar stabilization exercise and sling exercise on lumbosacral region angle, lumbar muscle strength, and pain scale of patients with chronic low back pain. Journal of Physical Therapy Science. 2018;30(1):18-22. doi: 10.1589/jpts.30.18.

8. Saragiotto BT, Maher CG, Yamato TP, Costa LO, Menezes Costa LC, Ostelo RW, Macedo LG. Motor control exercise for chronic non-specific low-back pain. Cochrane Database Syst Rev. 2016;(1):CD012004. doi: 10.1002/14651858. CD012004. PubMed PMID: 26742533.

9. Hall CM, Brody LT, Therapeutic exercise: moving toward function. Philadelphia: Lippincott Williams \& Wilkins; 2005.

10.Vezina MJ, Hubley-Kozey CL. Muscle activation in therapeutic exercises to improve trunk stability. Archives of Physical Medicine and Rehabilitation. 2000;81(10):1370-1379. doi: 10.1053/apmr.2000.16349.

11.Davidson KL, Hubley-Kozey CL. Trunk muscle responses to demands of an exercise progression to improve dynamic spinal stability. Archives of Physical Medicine and Rehabilitation. 2005;86(2):216-23. doi: 10.1016/j. apmr.2004.04.029.

12.Sahrmann S. Diagnosis and treatment of movement impairment syndromes. Elsevier Health Sciences; 2001.

13.Lee JK, Desmoulin GT, Khan AH, Park EJ. Comparison of 3D spinal motions during stairclimbing between individuals with and without low back pain. Gait \& Posture. 2011;34(2):2226. doi: 10.1016/j.gaitpost.2011.05.002.

14.Müller R, Ertelt T, Blickhan R. Low back pain affects trunk as well as lower limb movements during walking and running. Journal of Biomechanics. 2015;48(6):1009-14. doi: 10.1016/j.jbiomech.2015.01.042.

15.Lamoth CJ, Meijer OG, Wuisman PI, Van Dieën JH, Levin MF, Beek PJ. Pelvis-thorax coordination in the transverse plane during 
walking in persons with nonspecific low back pain. Spine. 2002;27(4):E92-9.

16. Taniguchi M, Tateuchi H, Ibuki S, Ichihashi N. Relative mobility of the pelvis and spine during trunk axial rotation in chronic low back pain patients: A case-control study. PloS One. 2017;12(10):e0186369. doi: 10.1371/journal. pone.0186369. PubMed PMID: 29040298. PubMed PMCID: PMC5645112.

17.Scholtes SA, Gombatto SP, Van Dillen LR. Differences in lumbopelvic motion between people with and people without low back pain during two lower limb movement tests. Clinical Biomechanics. 2009;24(1):7-12. doi: 10.1016/j.clinbiomech.2008.09.008.

18.Gombatto SP, Collins DR, Sahrmann SA, Engsberg JR, Van Dillen LR. Gender differences in pattern of hip and lumbopelvic rotation in people with low back pain. Clinical Biomechanics. 2006;21(3):263-71. doi: 10.1016/j. clinbiomech.2005.11.002.

19.Shojaei I, Salt EG, Hooker Q, Van Dillen LR, Bazrgari B. Comparison of lumbo-pelvic kinematics during trunk forward bending and backward return between patients with acute low back pain and asymptomatic controls. Clinical Biomechanics. 2017;41:66-71. doi: 10.1016/j.clinbiomech.2016.12.005.

20.Hubley-Kozey CL, Vezina MJ. Differentiating temporal electromyographic waveforms between those with chronic low back pain and healthy controls. Clinical Biomechanics. 2002;17(9-10):621-9. doi: 10.1016/S02680033(02)00103-1.

21. Hubley-Kozey CL, Vezina MJ. Muscle activation during exercises to improve trunk stability in men with low back pain. Archives of Physical Medicine and Rehabilitation. 2002;83(8):11008. doi: 10.1053/apmr.2002.33063.

22. Hubley-Kozey CL, Hanada EY, Gordon S, Kozey J, McKeon M. Differences in abdominal muscle activation patterns of younger and older adults performing an asymmetric legloading task. PM\&R. 200;1(11):1004-13. doi: 10.1016/j.pmrj.2009.09.018.

23. Hubley-Kozey CL, Hatfield GL, Davidson KC. Temporal coactivation of abdominal muscles during dynamic stability exercises.
The Journal of Strength \& Conditioning Research. 2010;24(5):1246-55. doi: 10.1519/ JSC.0b013e3181ce24c7.

24.Moreside JM, Quirk DA, Hubley-Kozey CL. Temporal Patterns of the Trunk Muscles Remain Altered in a Low Back-Injured Population Despite Subjective Reports of Recovery. Archives of Physical Medicine and Rehabilitation. 2014;95(4):686-98. doi: 10.1016/j. apmr.2013.10.003.

25. Manchikanti L. Epidemiology of low back pain. Pain Physician. 2000;3(2):167-192.

26. Nachemson AL, Schultz AB, Berkson MH. Mechanical Properties of Human Lumbar Spine Motion Segments: Influences of Age, Sex, Disc Level, and Degeneration. Spine.1979;4(1):1-8.

27.Sullivan MS, Dickinson CE, Troup JD. The influence of age and gender on lumbar spine sagittal plane range of motion. A study of 1126 healthy subjects. Spine. 1994;19(6):682-86. doi: 10.1097/00007632-199403001-00007. PubMed PMID: 8009333.

28.Shojaei I, Allen-Bryant K, Bazrgari B. Viscoelastic response of the human lower back to passive flexion: the effects of age. Annals of Biomedical Engineering. 2016;44(9):2817-26. doi: 10.1007/s10439-016-1569-7.

29.Airaksinen 0, Brox Jl, Cedraschi C, Hildebrandt J, Klaber-Moffett J, Kovacs F, Mannion AF, Reis S, Staal JB, Ursin H, Zanoli G. European guidelines for the management of chronic nonspecific low back pain. European Spine Journal. 2006;15(Suppl 2):s192. doi: 10.1007/s00586-006-1072-1. PubMed PMID: 16550448. PubMed PMCID: PMC3454542.

30.Tracey I, Bushnell MC. How neuroimaging studies have challenged us to rethink: is chronic pain a disease? The Journal of Pain. 2009;10(11):1113-20. doi: 10.1016/j. jpain.2009.09.001.

31.Shojaei I, Vazirian M, Croft E, Nussbaum MA, Bazrgari B. Age related differences in mechanical demands imposed on the lower back by manual material handling tasks. Journal of Biomechanics. 2016;49(6):896-903. doi: 10.1016/j.jbiomech.2015.10.037.

32.Wand BM, Parkitny L, O'Connell NE, Luomajoki H, McAuley JH, Thacker M, Moseley GL. 
Cortical changes in chronic low back pain: current state of the art and implications for clinical practice. Manual Therapy. 2011;16(1):1520. doi: 10.1016/j.math.2010.06.008.

33. Tsao H, Galea MP, Hodges PW. Driving plasticity in the motor cortex in recurrent low back pain. European Journal of Pain. 2010;14(8):832-9. doi: 10.1016/j.ejpain.2010.01.001.

34.Grachev ID, Fredrickson BE, Apkarian AV. Ab- normal brain chemistry in chronic back pain: an in vivo proton magnetic resonance spectroscopy study. Pain. 2000;89(1):7-18. doi: 10.1016/S0304-3959(00)00340-7.

35.Elia DS, Bohannon RW, Cameron D, Albro RG. Dynamic pelvic stabilization during hip flexion: a comparison study. Journal of Orthopaedic \& Sports Physical Therapy. 1996;24(1):30-6. doi: 10.2519/jospt.1996.24.1.30. 